\title{
La modalidad B-learning como alternativa de un ambiente de aprendizaje innovador
}

\author{
Blended learning as an alternative to an innovative learning environment \\ O modo B-aprendizagem, alternativamente, uma ambiente de aprendizagem \\ inovador
}

DOI: http://dx.doi.org/10.23913/reci.v6i11.60

René Rodríguez Zamora

Universidad Autónoma de Sinaloa, México

rene.rodriguez@info.uas.edu.mx

Leonor Antonia Espinoza Núñez

Universidad Autónoma de Sinaloa, México

leonor_espinoza63@hotmail.com

\section{Resumen}

En este artículo se presenta un estudio a partir del cual se definen las características con las que cuenta la modalidad B-Learning situándola como una alternativa innovadora para responder a las principales necesidades de un programa educativo presencial que permitan ofrecer mejores condiciones y más oportunidades para el aprendizaje. Se constituye así un análisis descriptivo basado en técnicas cuantitativas y cualitativas, orientado a justificar la pertinencia de la propuesta de implementación de esta modalidad en un programa presencial.

En un primer momento se hace una descripción de las herramientas tecnológicas existentes para la educación a distancia, se ubicaron como algunas de las más representativas: Claroline, Atutor, Dokeos, y Moodle. Identificado Moodle como la más idónea en cuanto a las condiciones funcionales para la implementación. Se elaboró posteriormente un diagnóstico del contexto escolar en lo referente a la calidad del servicio educativo y a las necesidades y requerimientos 
para la implementación de un programa académico. Encontrando que los estudiantes perciben que se les brinda un buen servicio educativo, sin embargo no se sienten del todo satisfechos con el desarrollo de la labor docente. Así también a pesar de que la institución cuenta con espacios grandes, éstos son insuficientes en cantidad, lo que ocasiona que se utilicen otros espacios como aulas improvisadas. Se manifiesta un uso desorientado que algunos profesores dan a los materiales didácticos y herramientas tecnológicas para el aprendizaje. Finalmente a partir de la descripción y el diagnóstico se definieron las características que debe conjugar el diseño de una propuesta que integre la modalidad B-Learning a un programa educativo, destacando las ventajas que esto podría ofrecer en lo que se refiere a la optimización en el uso de recursos y espacios, además de la posibilidad de poder ampliar la cobertura educativa.

Palabras clave: Educación a distancia, Gestor de Contenidos Educativos, B-Learning, Ambiente de Aprendizaje, Servicio Educativo.

\section{Abstract}

This article presents a study that defines the characteristics of B-Learning placing it as an innovative alternative to meet the main needs of a classroom education program to provide better conditions and more opportunities for learning. Thus constitutes a descriptive analysis based on quantitative and qualitative techniques, aimed to justify the relevance of the proposal's implementation of this method in a face-to-face program.

It first makes a description of the existing technological tools for distance education, some of the most representative: Claroline, Atutor and Dokeos, Moodle. Identifying Moodle as the most suitable in terms of functional conditions for implementation. It is subsequently made a diagnosis of the school context in relation to the quality of the education service and to the needs and requirements for the implementation of an academic program. Finding that students perceive that they are given a good educational service, however do not feel entirely satisfied with the development of teaching. So while the institution has large spaces, these are insufficient in quantity, what causes that they are used in other areas such as makeshift classrooms. Manifests a disoriented use that some teachers teaching materials and technological tools for learning. Finally from the description and diagnosis were defined the characteristics that must combine the design 
of a proposal that integrates the mode B-Learning to a curriculum stressing the advantages that this could offer in regards to the optimization in the use of resources and spaces, as well as the possibility to expand educational coverage.

Key words: distance education, Educational Content Management System, B-Learning, Blended learning, learning environment, educational service.

\section{Resumo}

Este artigo apresenta um estudo a partir do qual os recursos com que conta o modo B-Learning situando-o como uma alternativa inovadora para atender às principais necessidades de um programa de educação em sala de aula que permitirão proporcionar melhores condições e mais oportunidades para definir o aprendendo. Assim, constitui uma análise descritiva com base em técnicas quantitativas e qualitativas que visam justificar a relevância da proposta de implementação desta modalidade em um programa de sala de aula.

No começo uma descrição distância existente ferramentas de tecnologia da educação é feito, eles classificaram como alguns dos mais representativos: Claroline, ATutor, Dokeos e Moodle. Moodle identificado como o mais adequado em termos de condições funcionais para implementação. uma avaliação do contexto escolar é então desenvolvido em termos da qualidade da educação e as necessidades e os requisitos para a execução de um programa acadêmico. Encontrar que os alunos percebem que eles recebem um bom serviço educativo, no entanto eles não se sentem totalmente satisfeito com o desenvolvimento do ensino. Bem, mesmo que a instituição tem grandes espaços, estes são insuficientes em quantidade, causando outros espaços usados como salas de aula improvisadas. um uso desorientado alguns professores dão materiais educativos e ferramentas tecnológicas para os manifestos de aprendizagem. Finalmente a partir da descrição e dos recursos de diagnóstico que deve combinar desenhar uma proposta que integra o modo B-Learning para um programa educacional, destacando as vantagens que pode oferecer no que diz respeito à otimização definiu o uso de recursos e espaços, bem como a possibilidade de expandir a cobertura educacional.

Palavras-chave: Educação a Distância, Educação Content Manager, B-Learning, ambiente de aprendizagem, serviço educativo. 


\section{Introducción}

El reto que presentan las Universidades hoy en día es el aumento en la demanda de ingreso. El Incremento de estudiantes que egresan de las instituciones de nivel medio superior provoca que el número de estudiantes rechazados para ingresar a una Universidad Pública crezca.

De acuerdo con la ANUIES (Asociación Nacional de Universidades e instituciones de Educación Superior), en México existen más de trecientas universidades públicas (ANUIES, 2013), cabe mencionar que son las universidades que se han afiliado a esta asociación las cuales ofrecen diversas modalidades en la lógica de responder a las demandas de cobertura.

Esta situación sube año con año, cada vez son más los estudiantes que terminan sus estudios de nivel medio superior y tienen como primera opción ingresar a una Universidad Pública para continuar sus estudios.

El motivo por el cual sucede esto es por el espacio y la infraestructura insuficientes con que cuentan actualmente las instituciones públicas, ya que al no contar con dichos espacios, su capacidad de dar el servicio educativo a los estudiantes es limitado. Otra condición que también afecta es el financiamiento insuficiente que reciben las instituciones, esto es determinante debido a que sin el presupuesto suficiente, no se pueden habilitar mayores espacios o crecer en infraestructura para brindar un mejor servicio.

En este orden de ideas, la educación a distancia surge como una alternativa viable de intervención. Esta modalidad permite ofrecer entornos de aprendizaje mediante métodos, técnicas, estrategias y medios, en la que el profesor y el alumno se encuentran separados físicamente y solo se relacionan de manera presencial ocasionalmente.

El uso del modelo de educación a distancia, tanto en la modalidad E-Learning como en la modalidad B-Learning se ha impulsado en los últimos años con dos propósitos principales: ofrecer una opción más que permita ampliar la cobertura para que más estudiantes tengan la 
oportunidad de seguir sus estudios y con esto disminuir el número de aspirantes rechazados; y por otro lado, complementar los procesos de enseñanza y aprendizaje de los programas tanto escolarizados como semiescolarizados.

Se han realizado trabajos de investigación donde se ha implementado esta modalidad, obteniendo buenos resultados, como el que realizaron Mariño y Lopez (2007) donde describen la experiencia de haber implementado el modelo B-learning para la asignatura de Modelos y Simulación del programa de licenciatura en Sistemas de la Facultad de Ciencias Exactas y Naturales y Agrimensura de la Universidad Nacional del Nordeste en Argentina.

Si bien es cierto las investigaciones relacionadas con la implementación de las modalidades de educación a distancia aportan elementos importantes para el desarrollo de un programa académico, no abordan el aspecto de la optimización en el uso de recursos como son: costos de operación, ahorro de inversión en infraestructura, recurso humano y tiempo. Esto tiene que ver con sacar el mayor provecho a los recursos con los que cuenta una Institución y el dar una buena calidad en el servicio educativo a los estudiantes, utilizando dichos recursos de la mejor forma posible, obteniendo con ello buenos resultados y mayores beneficios.

El modelo B-Learning es un modelo de enseñanza y aprendizaje que combina las sesiones en modalidad presencial con las sesiones no presenciales, a diferencia de la modalidad E-Learning, la cual se oriente primordialmente a las sesiones no presenciales.

La modalidad B-Learning es considerada por varios autores como una opción viable para la educación. Fainholc (2006) considera que la modalidad B-Learning es "una estrategia de formación más autogestionaria y autónoma por parte del estudiante como también más cercana y pertinente respecto de una aproximación individual y grupal en la construcción de conocimiento". Vera (2008) argumenta que es una interesante estrategia ya que integra las mejores prácticas pedagógicas con lo último en tecnología para entornos virtuales de aprendizaje, además de extender la oferta educativa, mejorar la interacción entre los miembros de una comunidad, y aumentar la motivación de los estudiantes, entre otras cosas.

Considerando lo anterior, el presente artículo tiene como objetivo mostrar la modalidad $B$ Learning como una alternativa en la selección de los medios adecuados para la necesidad educativa, ya que se trata de un modelo eclético que está compuesto por métodos utilizados en la 
educación presencial y funcionalidades del aprendizaje electrónico o E-Learning, buscando con esto mejorar las fortalezas y disminuir las limitaciones de ambas modalidades y fomentar la participación de los estudiantes como responsables de su propio aprendizaje.

\section{MÉTODO}

Para la aplicación del estudio de campo se tomó como segmentos poblacionales a estudiantes de la carrera de ingeniería en sistemas computacionales del Instituto Tecnológico de Mazatlán Unidad II en el estado de Sinaloa, México, jóvenes de 18 a 23 años de edad, considerando el total de la población conformada para 140 estudiantes, ya que el diseño de la propuesta está orientado al programa educativo que atiende a esa población.

Para la recolección de datos se diseñó un cuestionario a partir de tres unidades de análisis principales: El uso de la plataforma Web, la calidad del servicio educativo y las necesidades de la institución. Este instrumento de recolección de datos fue validado aplicando el coeficiente Alfa de Cronbach. El cuestionario está compuestos por 19 items abordando los principales atributos de las variables a medir.

El análisis de los datos recolectados es procesado con medidas de tendencia central, frecuencia y porcentajes para la descripción de las características bajo las cuales puede ser implementada con eficiencia y pertinencia la modalidad propuesta. Por otra parte, se consideran las necesidades detectadas para el diseño de una plataforma en la modaldad B-learning conducente a la optimización de recursos y la mejora de los procesos de aprendizaje.

\section{HERRAMIENTAS TECNOLÓGICAS PARA LA EDUCACIÓN A DISTANCIA}

Roquet (2006) define la educación a distancia como "un sistema o modalidad educativa en que uno o varios estudiantes se encuentran geográficamente separados de un centro de enseñanza y del docente, es decir, ambos no se encuentran en el mismo espacio físico, por lo que hay un distancia espacial entre los dos, lo que determina que estos interlocutores para comunicarse tienen que hacerlo utilizando medios que salven esa distancia. Esta circunstancia hace que se practique un aprendizaje flexible y autónomo, que haya una comunicación personalizada y un uso permanente de materiales didácticos, que son elaborados por un grupo de expertos apoyados por una administración institucional." 
La educación a distancia ha estado evolucionando desde que inició con la enseñanza por correspondencia, pasando por tres etapas; la primera etapa se caracteriza por el desarrollo de textos y manuales impresos, los cuales eran enviados por correspondencia; la segunda etapa hace referencia a la integración de los materiales audiovisuales, que consistían en diapositivas, audiocasete, videocasete, entre otros, además de los materiales impresos; la tercera etapa es la integración de las dos anteriores pero de manera digital, es aquí donde entran las Tecnologías de la Información y Comunicación (TIC) a revolucionar la educación a distancia integrándola a la era digital.

El uso de las TIC's en la educación ha logrado grandes cambios, debido a que los métodos de enseñanza están siendo alcanzados por la tecnología, ya que el acceso a la información es más fácil ahora que hace décadas, además que existe una inmensa cantidad disponible en la red.

Para Roquet (2006) la era digital en la educación a distancia se caracteriza por la integración de la enseñanza por correspondencia y la enseñanza multimedia a través de las TIC's, donde los materiales didácticos dejan de ser entregados de forma tangible (Impresos en papel, audiocasetes videocasetes, diapositivas, etc) y son entregados de manera digital o virtual.

A esta etapa García (2002) también le define como campus virtual o enseñanza virtual, basada en la enseñanza por medio de redes de computadoras, donde todos los usuarios pueden interactuar entre sí, ya sea de manera síncrona o asíncrona. Además éstos pueden tener los materiales de los módulos disponibles a cualquier hora, o aplicar un examen de evaluación a cualquier hora y enviarla de manera electrónica en ese momento.

Es aquí donde se hace hincapié a dos modalidades de educación a distancia basadas en las TIC's, que fueron las que revolucionaron el concepto de enseñanza a distancia y que hoy en día son las más utilizadas tanto en Universidades como Empresas y Corporaciones, estas son: la modalidad E-Learning y la modalidad B-Learning.

\section{PLATAFORMAS EXISTENTES}

Una plataforma se puede definir como un sitio web donde los usuarios, en este caso jóvenes estudiantes, pueden comunicarse entre sí, ofreciendo medios como foros, chat, video conferencias, compartir archivos, etc. Normalmente para acceder a este tipo de sitios se requiere que los usuarios se autentifiquen para que puedan tener distintos perfiles. Existen diversas 
plataformas en internet como Claroline, Atutor, DOKEOS y MOODLE, las cuales se utilizan para la creación y planificación de contenidos curriculares, además de dar la atención a la comunidad educativa en internet.

\section{A. Claroline}

El proyecto Claroline (Universidad Católica de Lovaina) inicio en el año 2000, por parte del Instituto Pedagógico Universitario de Multimedia de la Universidad Católica de Lovain en Bélgica, iniciado por Thomas De Preatere, Hugues Peeters y Christophe Gesché. Claroline (ver Figura 1) es un software creado con código abierto y distribuida bajo Licencia GPL, permitiendo implementar una plataforma para el aprendizaje y la colaboración en línea, permite crear espacios para los cursos, este software está disponible en varios idiomas y se puede descargar gratuitamente e instalar libremente. Claroline es multiplataforma ya que está basado en PHP, MySQL y Apache, se puede instalar en Windows, Linux o MAC.

Figura 1. Plataforma "Claroline".

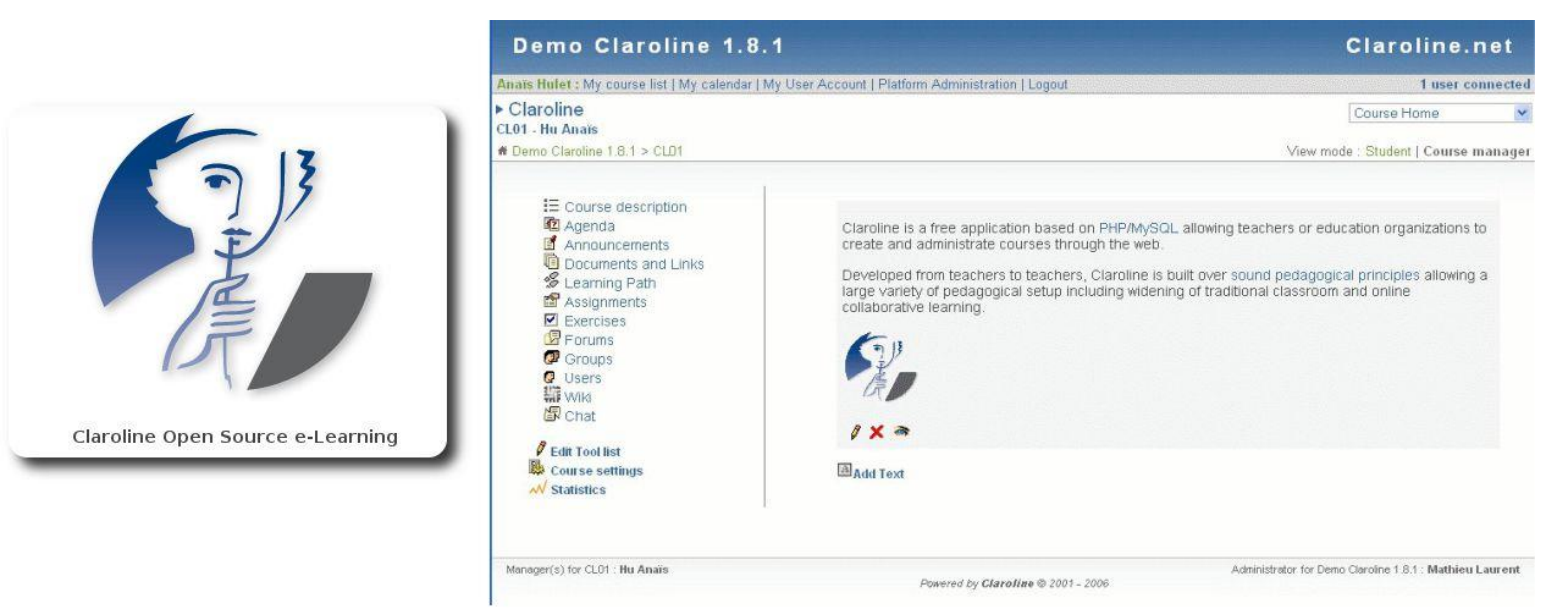

Fuente: Sitio web http://www.claroline.net/

La plataforma es fácil de instalar y usar, permite una flexibilidad para personalizarla y crear estilos de acuerdo a las necesidades del usuario, permite al profesor el disponer todas las herramientas necesarias para la creación y organización de los cursos. El inconveniente es que no tiene la opción de exportar los cursos, es poco modificable, cuenta con pocos módulos y plugins para descargar y su personalización es un tanto dificultosa. 


\section{B. ATUTOR}

ATutor (2002) pertenece al grupo de los Gestores de Contenidos de Aprendizaje (LCMS, por sus siglas en inglés Learning Content Management System). También constituye un entorno de red social de código abierto. Se distribuye gratuitamente como software libre bajo licencia GNU, ha sido desarrollado y actualizado en sus diferentes versiones por el Centro Adaptativo de Recursos Tecnológicos (ATRC) de la Facultad de Información de la Universidad de Toronto.

Figura 2. Plataforma "ATutor".

\section{ATutor $^{\oplus}$}

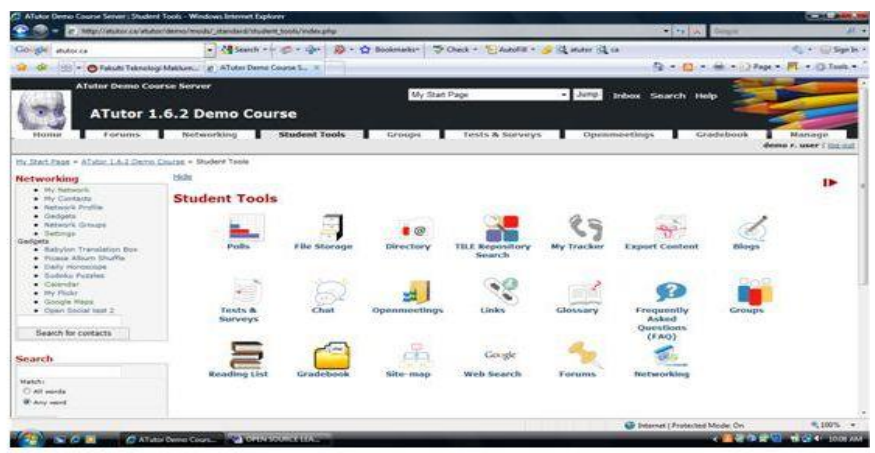

Fuente: Sitio web http://www.atutor.ca/

ATutor ha sido diseñado también con el objetivo de lograr accesibilidad y adaptabilidad para personas con algún tipo de discapacidad. Desde el punto de vista técnico, ATutor está basado en PHP, MySQL y Apache, el sistema es multiplataforma y funciona en cualquier sistema operativo Linux/BSD/Unix, Windows y MAC.

\section{DOKEOS}

Dokeos (2010) pertenece al grupo de los Gestores de Contenidos Educativos (LMS, por sus siglas en inglés Learning Management System). Es una plataforma E-Learning que permite a los docentes y alumnos tener acceso a funcionalidades para desarrollar actividades administrativas y académicas. Dokeos reúne e integra todos los componentes necesarios para la gestión, comunicación, evaluación y seguimiento de actividades de enseñanza y aprendizaje en un espacio virtual. 
Figura 3. Sitio Web Dokeos.
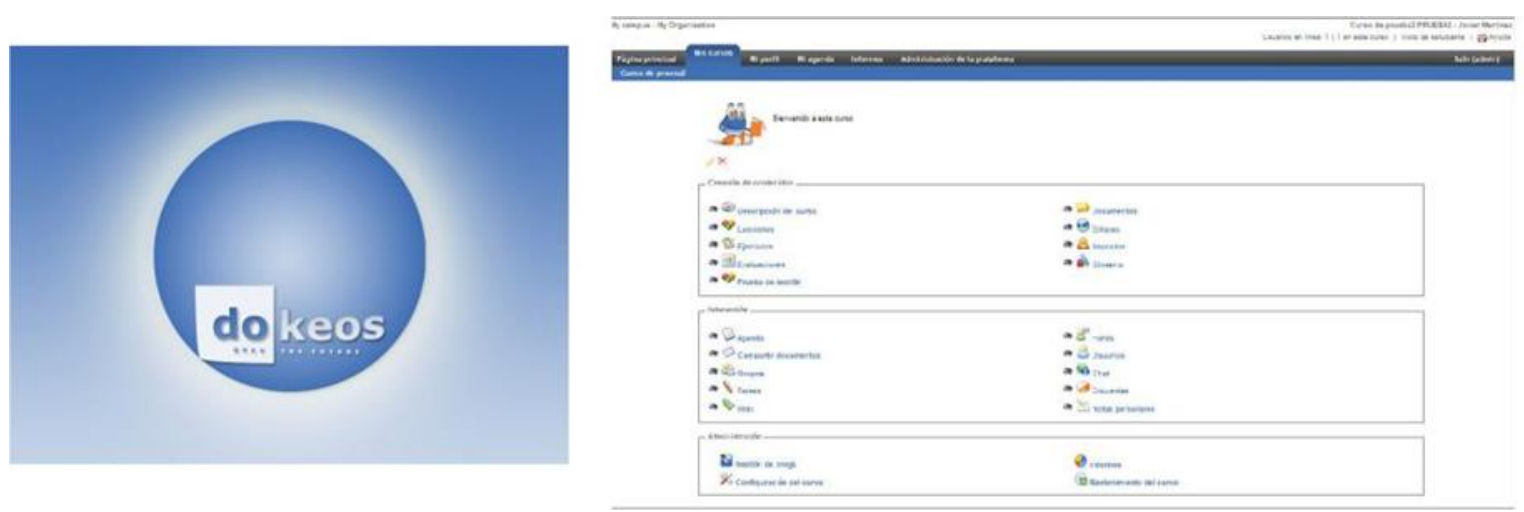

Fuente: Sitio web https://www.dokeos.com/

Dokeos (ver Figura 3) permite que los profesores puedan acceder con facilidad a las prestaciones del sistema, administrar sus documentos en procesador de texto, pdf, gráficos, etc., sin necesidad de llevar a cabo un tratamiento informático especial.

Esta plataforma permite integrar múltiples fuentes de información multimediales: video, audio, y voz. Requiere, como las otras, de una conexión a Internet que puede ser dial-up o dedicada. El programa cliente es un navegador común (Internet Explorer, Chrome, Firefox, etc.). El sistema Dokeos está desarrollado en el lenguaje PHP y HTML, usando como gestor de base de datos MySQL sobre un sistema operativo multiusuario y un servidor Web Apache. Dokeos no es un software libre como otros sistemas dedicados a la gestión de contenidos para el aprendizaje (Claroline, ATutor o Moodle), este ofrece el servicio del aula virtual por un costo de rentabilidad.

\section{MOODLE}

Moodle (Module Object-Oriented Dynamic Learning Environment) (Dougiamas, 2001) es una aplicación que pertenece también al grupo de los Gestores de Contenidos Educativos. Esta plataforma (ver Figura 4) permite la creación de cursos y sitios web, con esto un centro educativo, institución o empresa, puede gestionar recursos educativos proporcionados por docentes y organizar el acceso a estos para los estudiantes, permitiendo también la comunicación entre ambos. 
Figura 4. Plataforma web Moodle.

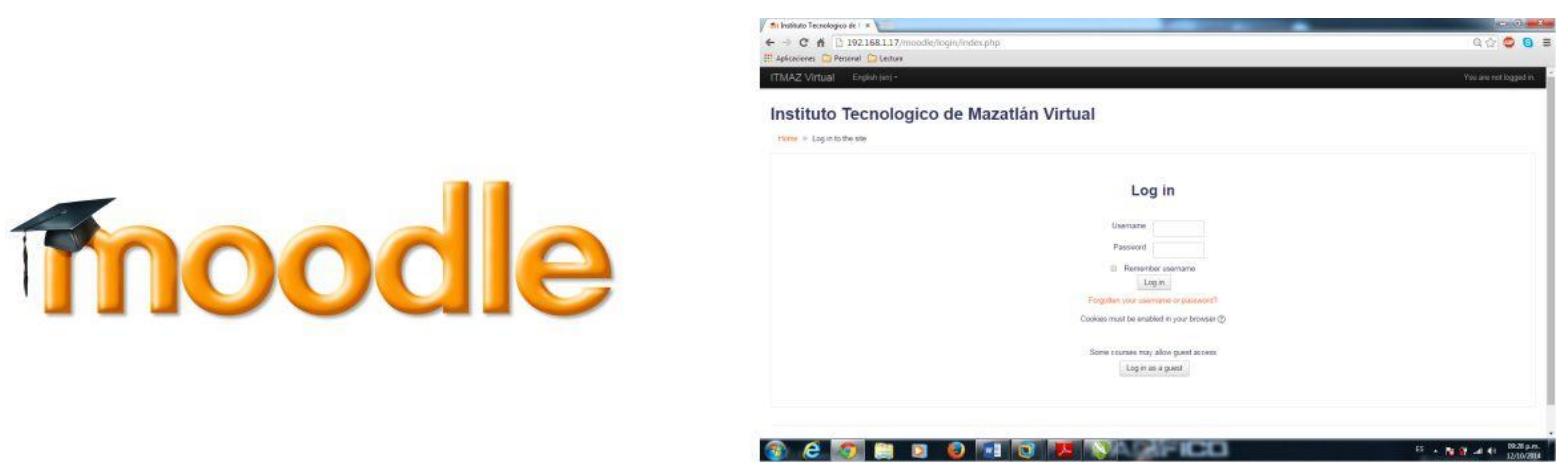

Fuente: Sitio web https://moodle.org/

Moodle está diseñada y desarrollada basándose en la filosofía del aprendizaje denominada pedagogía constructivista social, la cual incluye cuatro conceptos subyacentes: Constructivismo, Construccionismo, Constructivismo Social y Aprendizaje Separado y/o conectado.

Moodle es quizás la plataforma que ha mantenido la línea evolutiva más consistente. Esto se debe en buena medida al crecimiento de la comunidad de usuarios que dan soporte a este sistema. (Ver Figura 5).

Figura 5. Evolución de Moodle.
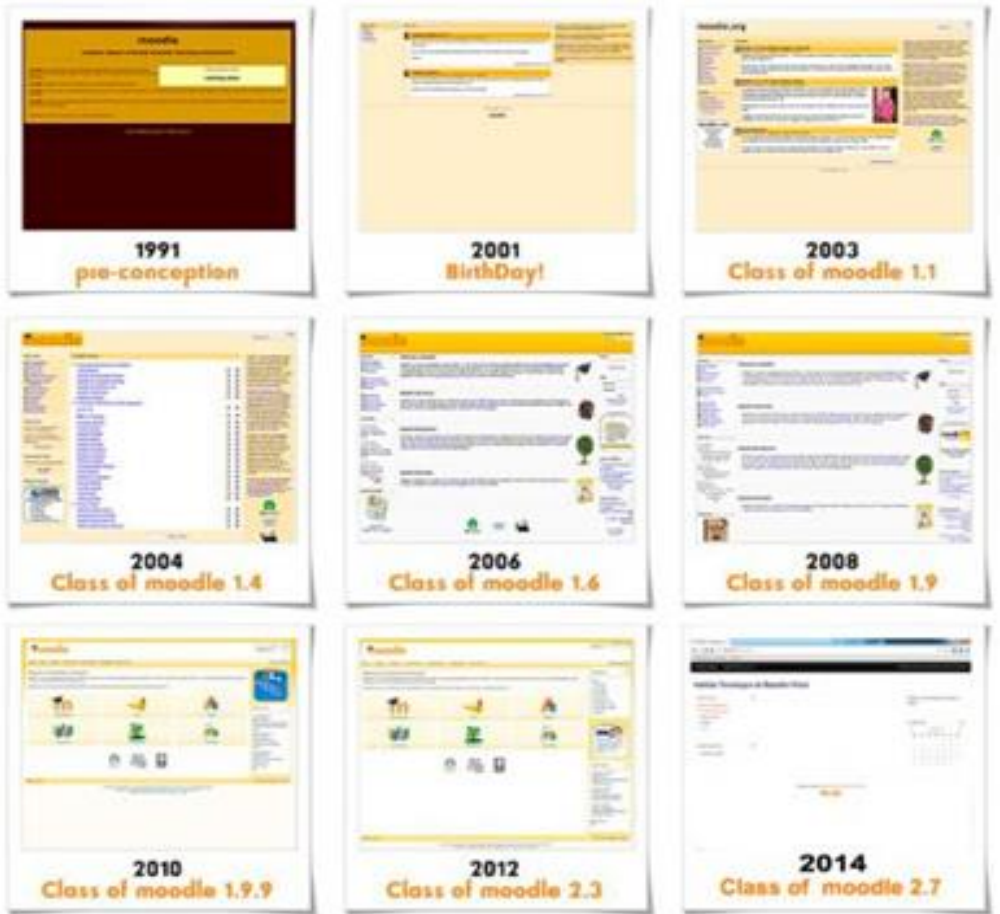

Fuente: Sitio web https://moodle.org/ 
Existen diversas herramientas tecnológicas para desarrollar la educación a distancia. Estas herramientas se concentran fundamentalmente en plataformas software constituidas como sistemas gestores de contenidos, particularmente en lo que se refiere a contenidos educativos. Las plataformas presentadas en esta sección comparten características funcionales que las convierten en opciones viables para la implementación de modalidades E-Learning y/o B-Learning. De acuerdo a la descripción de algunas de las características técnicas básicas de estas plataformas, existen condiciones que tienen que formar parte del contexto de un programa educativo en cuanto a la infraestructura y la planeación de los servicios que brinda una institución

\section{RESULTADOS.}

\section{CONTEXTO DEL PROGRAMA EDUCATIVO}

Para que una propuesta innovadora en el aprendizaje pueda ser exitosa requiere de una serie de condiciones que cambien la perspectiva de la educación tradicional, donde se rompan los esquemas cerrados y lineales de control de la administración escolar. Para Malbernat (2008) las instituciones educativas deben de replantear los momentos en el aula, la infraestructura tecnológica que ponen a disposición de los docentes, y los recursos educativos que se entregan para estimular a los estudiantes. Casas Armengol (2005) menciona que el problema radica en las estructuras rígidas y centralizadas que generan dificultades para la implementación de los cambios innovadores. Es importante se tome en cuenta el perfil del estudiante que muestra en los resultados un determinado nivel de dominio digital en el uso de las plataformas web y la facilidad de acceso a una computadora y/o dispositivo móvil con conexión a internet. A este respecto como se muestra en la Tabla 1 existe una disposición y experiencia aceptable en el manejo de las plataformas así como acceso a una computadora o dispositivo móvil.

Tabla 1. Facilidad de acceso a medios digitales.

\begin{tabular}{|c|c|c|c|c|c|c|c|}
\hline Item & $\mathbf{N}$ & Mínimo & Máximo & Media & $\mathbf{X x}$ & Ls & $\mathbf{L i}$ \\
\hline $\begin{array}{c}\text { Fácil acceso a una computadora } \\
\text { y/o dispositivo móvil }\end{array}$ & 140 & 3 & 10 & 9.23 & \multirow{4}{*}{8.93} & \multirow{4}{*}{9.32} & \multirow{4}{*}{8.55} \\
\hline Facilidad de acceso a internet & 140 & 1 & 10 & 8.70 & & & \\
\hline $\begin{array}{c}\text { Uso de la computadora para } \\
\text { trabajos escolares }\end{array}$ & 140 & 1 & 10 & 9.05 & & & \\
\hline Navegación en internet & 140 & 1 & 10 & 9.30 & & & \\
\hline
\end{tabular}

Fuente: Elaboración propia 
De acuerdo a los resultados, considerando los valores de la $X x$ y su respectivo límite inferior y superior, se infiere que a los estudiantes se les facilita el tener acceso a una computadora y/o dispositivo móvil, además de darle el uso adecuado para la realización de trabajos escolares y navegar por internet, pero se les dificulta el tener una conexión de acceso.

Es decir, existe la disposición por parte del estudiante a adentrarse al uso de estrategias digitales como herramientas de aprendizaje, sin embargo no cuentan con todas las condiciones para su aprovechamiento.

La implementación de propuestas innovadoras como la educación a distancia exige que se haga frente al escepticismo que genera la falta de confianza por el desconocimiento de las ventajas que ofrecen las herramientas tecnológicas de las que se puede hacer uso, pero para ello se requiere que se generen las condiciones adecuadas que incluyen adaptaciones que van desde el modelo educativo, pasando por la infraestructura física y tecnológica, hasta llegar a una coordinación con los servicios administrativos; ya que la falta de congruencia entre los requerimientos de este tipo de propuestas y los servicios ofrecidos, lo que puede generar insatisfacción que resulta en el demérito de la calidad del servicio educativo.

La calidad del servicio educativo involucra aspectos como: disponibilidad de los docentes, que se puede ver reflejada en otros aspectos como la satisfacción de los estudiantes con respecto a su desempeño y a las relaciones que con él establece y que permiten mejorar el aprendizaje para el logro de competencias. De acuerdo a lo anterior, se muestran en los resultados obtenidos en la Tabla 2, que existen algunas variables que refieren a las características del contexto escolar que están por encima de $X x$, de esta manera se observa que el joven declara recibir buena calidad en el servicio educativo que ofrece la institución, los estudiantes se sienten también satisfechos del nivel de aprendizaje alcanzado y sienten que los logros en el desarrollo de las competencias establecidas en el programa han aumentado. Sin embargo, hay poca disponibilidad por parte de los docentes para asesoría o tutorías, además de hacer poco uso de los materiales didácticos disponibles para el aprendizaje de los estudiantes, ocasionando con ellos que no estén totalmente satisfechos con el desarrollo de la práctica docente en lo que se refiere al uso de medios digitales. 
Tabla 2. Calidad en el Servicio Educativo de la Institución.

\begin{tabular}{|c|c|c|c|c|c|c|c|}
\hline Item & $\mathbf{N}$ & Mínimo & Máximo & Media & $\mathbf{X x}$ & Ls & $\mathbf{L i}$ \\
\hline Calidad del Servicio Educativo & 140 & 1 & 10 & 8.30 & \multirow{7}{*}{7.91} & \multirow{7}{*}{8.14} & \multirow{7}{*}{7.68} \\
\hline Disponibilidad de los maestros & 140 & 1 & 10 & 7.83 & & & \\
\hline $\begin{array}{l}\text { Uso adecuado de los materiales } \\
\text { didácticos para el aprendizaje }\end{array}$ & 140 & 1 & 10 & 7.76 & & & \\
\hline $\begin{array}{l}\text { Satisfacción con el personal } \\
\text { docente }\end{array}$ & 140 & 1 & 10 & 7.74 & & & \\
\hline $\begin{array}{l}\text { Satisfacción con el desarrollo del } \\
\text { aprendizaje }\end{array}$ & 140 & 1 & 10 & 7.96 & & & \\
\hline $\begin{array}{l}\text { Interacción maestro-alumno a } \\
\text { través del uso de una plataforma }\end{array}$ & 140 & 1 & 10 & 7.68 & & & \\
\hline $\begin{array}{c}\text { Aumento del nivel de aprendizaje } \\
\text { conforme las competencias } \\
\text { establecidas en el programa }\end{array}$ & 140 & 1 & 10 & 8.11 & & & \\
\hline
\end{tabular}

Fuente: Elaboración propia

Otro aspecto importante que fue incluido a considerar como parte de un diagnóstico se refiere a las necesidades de la institución. En este sentido, como se muestra en la Tabla 3, existen variables que están por encima de $X x$, tomando en cuenta los respectivo límite superior e inferior se observa que los servicios y los recursos que ofrece la institución son aceptables y el acceso a las distintas fuentes de información son buenas, pero algunos de los espacios muestran poca aceptación para ser utilizados como aulas de clases.

Tabla 3. Encuesta sobre las necesidades de la institución.

\begin{tabular}{|c|c|c|c|c|c|c|c|}
\hline Item & $\mathbf{N}$ & Mínimo & Máximo & Media & Xx & Ls & Li \\
\hline Espacios adecuados para las clases & 140 & 1 & 10 & 7.93 & & & \\
\cline { 1 - 1 } $\begin{array}{c}\text { Recursos y servicios en tecnología } \\
\text { de la información que ofrece la } \\
\text { institución }\end{array}$ & 140 & 1 & 10 & 8.13 & 8.11 & 8.29 & 7.93 \\
\cline { 1 - 4 } $\begin{array}{c}\text { Acceso a las distintas fuentes de } \\
\text { información }\end{array}$ & 140 & 1 & 10 & 8.28 & & \\
\hline
\end{tabular}

Fuente: Elaboración propia

Con base en la información obtenida, se concluye que la institución a pesar de que tiene grandes espacios, éstos son insuficientes, lo que provoca que se utilicen otros que no necesariamente son los más adecuados para ser utilizados como aulas.

Por otro lado, a pesar de que los estudiantes tienen fácil acceso al uso de los dispositivos móviles o a una computadora con conexión a internet y cuentan con cierto conocimiento en el uso de plataformas virtuales para la enseñanza, el uso desorientado que dan algunos profesores a los materiales didácticos y herramientas tecnológicas para el aprendizaje ocasiona que se abra una 
brecha en la comunicación empática que debe existir entre profesor-estudiante para la construcción de los saberes.

\section{REQUERIMIENTOS PARA EL DISEÑO DE UNA PROPUESTA INTEGRADORA}

En la modalidad E-Learning la planificación es un requerimiento fundamental para asegurar la implementación de un programa educativo totalmente a distancia. La naturaleza de esta modalidad exige una organización sistemática y rigurosa que garantice el logro de objetivos. Las experiencias, crecimiento y diversificación de opciones en diferentes centros educativos son una muestra de la aceptación y funcionalidad que ha garantizado, abriendo cada vez más posibilidades de formación, capacitación y actualización. En el caso de la modalidad $B$ Learning, se presenta un escenario más complejo ya que hay que conjugar las características del contexto de un programa presencial con las de un programa a distancia, donde los roles aunque son diferentes, buscan amalgamarse para facilitar la incorporación de los estudiantes.

El carácter semipresencial requiere desde la planeación en la elaboración del diseño, partir de un encuadre coherente con un modelo educativo y un enfoque pedagógico que permita la institucionalización y evite el manejo disperso y el uso equivocado de las herramientas, requiere además de un profesor tutor habilitado que pueda servir de acompañamiento del estudiante tanto en el uso como en el sentido que se le da a la tecnología como parte de su proceso de formación. Requiere también de una adaptación a la visión que juega la relación maestro-alumno dentro de estos espacios.

La políticas educativas de una institución que ofrece un programa educativo presencial deben incluir de manera puntual en su definición la garantía de contar con una infraestructura adecuada que garantice la conectividad necesaria y el acceso eficiente a recursos tecnológicos que favorezca el diseño de estrategias de trabajo colaborativas dentro del contexto escolar.

Por otro lado, considerando alguna de las opciones de plataformas de ambientes de aprendizaje integrados como Dokeos o gestores de contenidos educativos como ATutor o Moodle, o alguna otra que se pudiera identificar, se debe hacer una selección que sea pertinente y acorde a las demandas del programa educativo que permita la formación del estudiante considerando las 
competencias requeridas en su perfil de egreso. Las estrategias didácticas o de aprendizaje contenidas en el diseño de la plataforma elegida deben de ser producto de un modelo educativo y de un enfoque pedagógico actual centrado en el aprendizaje donde el estudiante asume el rol principal al ser dotado de los medios necesarios para el aprendizaje autónomo y la metacognición, donde finalmente él planifica y controla su propio proceso como sujeto cognitivo.

\section{RECURSOS DE HARDWARE Y SOFTWARE UTILIZADOS}

Como recursos hardware, para el desarrollo de esta propuesta se utilizó un servidor HP ProLiant ML350 G6 con las siguientes características:

- 2 Procesadores Intel Xeon (2.40 Ghz/6 Core).

- 24 GB de memoria RAM y HDD de 3 TB.

- Windows Server 2008 R2.

También se plantea el uso de un equipo adicional habilitado como servidor para guardar la base de datos de la plataforma.

En la Figura 6 se muestra la propuesta de estructura de red local tanto alámbrica como inalámbrica que brinda el servicio de internet y conectividad al servidor para acceder a la plataforma.

Figura 6. Propuesta de estructura de red para la implementación de la modalidad B-Learning.
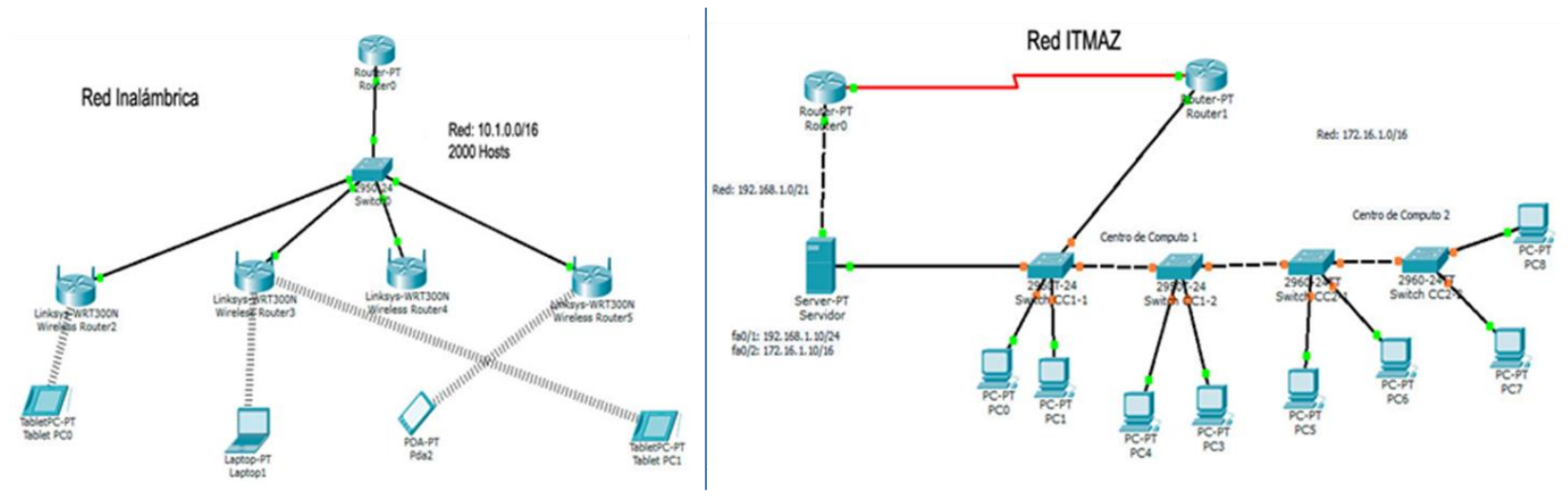

Fuente: Elaboración propia 
Como herramientas software se utilizó el gestor de máquinas virtuales VMware Workstation versión 10 para reservar espacio y recursos exclusivos para la plataforma. El sistema operativo que se instaló en la máquina virtual fue Linux Fedora 2.0 por razones de estabilidad y seguridad. Se instaló adicionalmente Apache y MySQL como servidores web de base de datos respectivamente.

Para la elaboración de la interfaz de usuario del servidor web a través de la cual se accede a la plataforma se utilizó el gestor de contenidos JOOMLA 3.3.6. Se configuró de acuerdo a las necesidades y normas de la institución. Como gestor de contenidos educativos se utilizó Moodle para construir la plataforma virtual para concretar la implementación de la modalidad $B$ Learning.

\section{DISCUSIÓN}

La modalidad B-Learning ha surgido como una alternativa para generar ambientes de aprendizaje innovadores donde se combina el rol presencial de alumnos y docentes en un contexto que se complementa con la acción autónoma de los estudiantes en su proceso de aprendizaje. Monsalve y Amaya (2014) lo denominan ambientes mixtos de aprendizaje, sin embargo como se muestra en los resultados el nivel del logro en habilidades tecnológicas exige la modificación sustancial de la práctica docente, lo que representa un verdadero reto pues ello conduce a diseñar estrategias y rutas más acordes con estos nuevos contextos de innovación; tanto el docente como el estudiante deben pensar en un aprendizaje basado en la interactividad, y las condiciones administrativas también deberán ir regulando nuevas formas de gestión escolar que priorice el otorgamiento de condiciones en cuanto a espacios y tiempos de aprendizaje que se adecúen a los nuevos perfiles.

Barberá (2008) señala ciertos componentes básicos que configuran el uso de estrategias digitales, entre los que enfatiza la planificación, la accesibilidad de la información, las herramientas de interacción y el seguimiento al desempeño como requisitos de carácter pedagógico a considerar al integrar el conjunto de herramientas tecnológicas. Si bien este estudio se limita a una población específica, muestra el acceso y la familiaridad con la que los jóvenes se adaptan y esperan de sus docentes mayores resultados en el aprovechamiento de las modalidades virtuales. El diseño de esta propuesta abre áreas de oportunidad ya que por una parte representa una estrategia a través 
de la cual se amplía la cobertura al mismo tiempo que se optimizan recursos en la gestión del servicio educativo.

Sin embargo, introducir las tecnologías en el campo de la educación exige la conformación de un principio pedagógico que debe estar en constante transformación, ya que así lo demanda en la acelerada era de la información en la que evidentemente el estudiante se ve involucrado, se habla entonces de aprendizajes significativos y autónomos como objetivo principal de la demanda curricular. Turpo (2013) habla de la convergencia en el B-learning para referirse a una conjunción de mediaciones pedagógicas y tecnológicas donde confluyen recursos para interactuar tanto en escenarios presenciales como en escenarios virtuales para el logro de una misma función u objetivo.

Se requiere desde este punto de vista un cambio de escenarios donde se parta de que el estudiante puede acceder de manera libre y autónoma al acceso de información, donde los espacios físicos dejen de verse como la única alternativa para la interacción que requiere el proceso de aprendizaje, donde se asuma una nueva forma de alfabetización que tenga su punto de partida en el acceso a los dispositivos al alcance para los jóvenes, y se entienda el conocimiento como un proceso de construcción social a partir de la colaboración.

\section{CONCLUSIONES}

La era digital en la educación a distancia se caracteriza por la integración de la enseñanza por correspondencia y la enseñanza multimedia a través de las tecnologías de la Información y comunicación, donde los materiales o recursos didácticos son proporcionados de manera digital, ello permite mayor cobertura de atención y mayor alcance para el usuario.

Los jóvenes estudiantes tienen fácil acceso a un dispositivo o computadora con conexión a internet, han desarrollado además cierta habilidad al uso de plataformas virtuales por lo que están conscientes del beneficio que puede representar como una herramienta de aprendizaje, sin embargo demandan de parte de sus profesores mayor orientación en el uso de estas herramientas y modalidades tecnológicas, lo que ocasiona que no se potencialice la interacción pedagógica entre docente y alumno. 
Las características de la modalidad B-Learning ofrecen un área de oportunidad para ayudar a mejorar la calidad educativa, también ofrecen la posibilidad de optimizar los recursos de infraestructura con los que cuenta una Institución y reducir a su vez los costos de operación, haciendo más factible los objetivos de un modelo y programa educativo.

Existe una gran diversidad de plataformas que pueden servir como medios en la construcción del aprendizaje, entre ellas ATutor, Claroline, Dokeos o Moodle, los criterios de selección de alguna de ellas por parte de una institución deben de ir más allá de las especificaciones técnicas o incluso del nivel de sofisticación que pueden proporcionar. Sin embargo estos criterios deben considerar una alineación entre las políticas institucionales, las capacidades de infraestructura física y tecnológica, y el nivel de habilitación tanto para el acceso como el uso por parte de la comunidad que forma parte de un programa educativo. Se debe tener un diagnóstico claro y objetivo de las condiciones reales de una institución para identificar las áreas de oportunidad que sirvan de puntos de referencia para definir y establecer medidas que ayuden a potencializar el uso de las herramientas tecnológicas en el aumento de las capacidades para generar ambientes de aprendizaje innovadores. 


\section{Bibliografía}

ANUIES. (2013). http://201.161.2.34/la_anuies/diries/.

Barberá, E. (2008). Aprender e-learning. Paidós, Barcelona.

Casas Armengol, M. (2005). Nueva universidad ante la sociedad del conocimiento. Revista de universidad y sociedad del conocimiento, 1-18.

Dokeos. (2010). DOKEOS eLearning made easy. Obtenido de Institut de Pédagogie universitaire et des Multimédias.

Dougiamas, M. (2001). Sitio web de Moodle. Recuperado el 2016, de http://moodle.org/

Fainholc, B. (2006). Optimizado las posibilidades de las TICs en Educación. Revista Electrónica de Tecnología Educativa, O(22), Recuperado de http://www.edutec.es/revista/index.php/edutec-e/article/view/502/236.

Garcia Aretio, L. (2002). La educación a distancia: De la teoria a la práctica. Ariel S.

Malbernat, L. R. (2008). Cambios institucionales para una nueva enseñanza en la educación superior. Revista de curriculum y formación del profesorado, 1-18.

Mariño, S. (2007). Aplicación del modelo b-learning en una asignatura "Modelos y Simulación" de la carrera de Sistemas de la FACENA- UNNE. Revista Electrónica de Tecnología Educativa., O(23), Recuperado de http://www.edutec.es/revista.

Monsalve Gómez, J. C. y Amaya Vanegas, D. M. (2014). Implementación de ambientes de aprendizaje b-learning: retos para docentes y estudiantes. Revista Colombiana de Ciencias Sociales, 5(2), 408-417.

Roquet García, G. (2006). Antecedentes historicos de la Educación a distancia. Mexico. México: UNAM.

Toronto, U. d. (2002). Sitio Web de Atutor. Obtenido de http://www.atutor.ca/

Turpo Gebera, O. (2013). Perspectiva de la convergencia pedagógica y tecnológica en la modalidad blended learning. RED, Revista de Educación a Distancia. Número 39. 
Universidad Católica de Lovaina, B. (2000). http://www.claroline.net/.

Vera, F. (2008). La modalidad Blended learning en la educación superior. Rancagua, Chile. 